\title{
A Lightweight Among Heavyweights
}

\section{Researchers have observed the lightest uranium isotope to date, offering insight into models of nuclear structure.}

\section{By Katherine Wright}

$\square$ iscovering new isotopes is like the stamp collecting of physics, but the consequences of adding to the set are much further reaching. A team of researchers using the Heavy Ion Research Facility in Lanzhou, China, has now expanded the collection with the discovery of the lightest uranium isotope to date [1]. The finding could have implications for our understanding of a particular type of radioactive decay that is still mysterious despite more than a century of work.

Uranium is an inherently unstable element. All of its isotopes are radioactive, with the most abundant ones having half-lives ranging from 150,000 to 4.5 billion years (roughly the age of Earth). Naturally occurring uranium contains between 140 and 146 neutrons. The newly discovered isotope has just 122, one fewer than the previous record for the element.

The team created the isotope in a "fusion-evaporation" reaction, which involved firing a beam of argon at a tungsten target and monitoring the fusion products. They identified two previously discovered light uranium isotopes-uranium-216

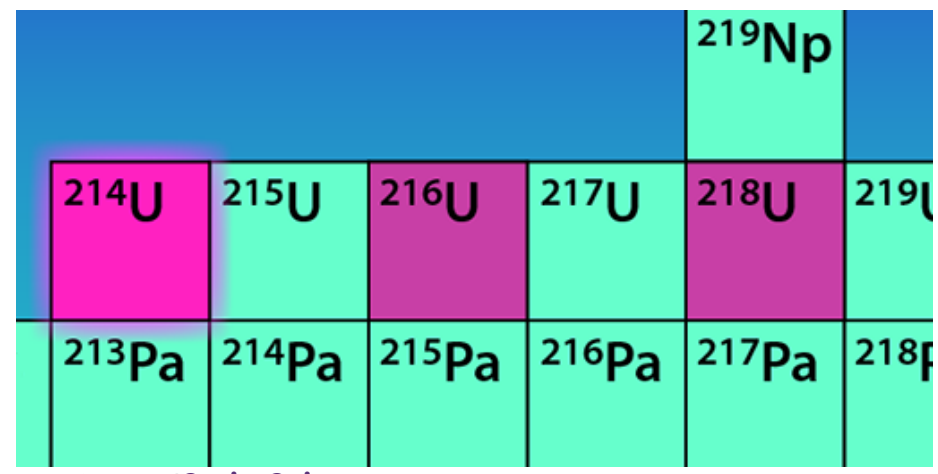

Credit: APS/Carin Cain and uranium-218-as well as the new one, uranium-214, which has a blink-and-you'll-miss-it half-life of $0.5 \mathrm{~ms}$.

The number of neutrons in this isotope sits near a so-called magic neutron number, specifically 126 , which makes it interesting for studying nuclear stability. Magic isotopes are unusually stable, and observing their near neighbors provides opportunities to probe how nuclear structure influences radioactive decay processes. In this case, measurements from the three observed uranium isotopes suggest that they experience an enhanced proton-neutron interaction compared with isotopes of other elements. This stronger interaction affects the formation of alpha particles in the nucleus, a complex quantum many-body problem whose details are still unknown.

Katherine Wright is the Deputy Editor of Physics.

\section{REFERENCES}

1. Z. Y. Zhang et al., "New $\alpha$-emitting isotope ${ }^{214} U$ and abnormal enhancement of $\alpha$-particle clustering in lightest uranium isotopes," Phys. Rev. Lett. 126, 152502 (2021). 